\title{
2006-943: TEACHING STUDENTS HOW TO EVALUATE THE REASONABLENESS OF STRUCTURAL ANALYSIS RESULTS
}

\section{James Hanson, Rose-Hulman Institute of Technology}

Dr. James Hanson is an Assistant Professor of Civil Engineering at the Rose-Hulman Institute of Technology. He teaches mechanics courses for the freshman through senior levels including structural analysis and design. He is a strong advocate of hands-on learning and problem-based learning. He is a licensed professional engineer. He has also taught at Cornell University and Bucknell University. 


\title{
Teaching Students How to Evaluate the Reasonableness of Structural Analysis Results
}

\begin{abstract}
Structural engineers, and engineers in general, depend heavily on software to assist in complex analyses of large problems. As the size and complexity of a problem increases, however, the potential for errors and the devastating impacts of those errors increase. Unfortunately, few faculty teach undergraduate students how to evaluate the reasonableness of their structural analysis results. Therefore, the National Science Foundation has funded a project to develop a version of undergraduate structural analysis course that teaches students to not only generate structural analysis results, but also to evaluate those results for reasonableness.
\end{abstract}

The author has interviewed practicing structural engineers to determine the methods they use to evaluate structural analysis results. The data from the interviews have been blended into a new version of the undergraduate structural analysis course. A comparison of syllabi from the old and new versions of the course shows that teaching evaluation of results can have minimal impact on the time spent on each topic on the syllabus.

The methods being incorporated into the new version of the course focus on simplifying situations into problems that can be easily solved and on anticipating features of complex solutions. This paper summarizes the methods incorporated in this course and provides several examples.

In exit interviews, students in both the old and new versions of the course expressed similar attitudes when asked about the existence of reasonable answers and the importance of evaluating results. Therefore, students already believed that reasonable answers can be obtained, and that it is important to evaluate their answers. However, when students taking both the old and new versions of the course were tested at the end of the course to measure their ability to evaluate the reasonableness of structural analysis results, the students in the new version of the course showed a measurable increase in ability to identify the most reasonable answer and to explain why it was the most reasonable answer.

\section{Introduction}

Categories of Errors

To help students learn methods for evaluation, the course begins with a description of four categories of errors in structural analysis and design: idealization of the real structure, assumptions inherent to the analysis method or design equations, roundoff error, and human error.

1. Idealization of the real structure. This category includes all of the assumptions we intentionally make in order to model a structure. Some examples include assuming unrestrained rotation at every joint of a truss, exactly straight members, or perfectly rigid diaphragms. Fortunately, many of the errors induced by the idealization of the structure have a relatively small impact. The load and strength reduction factors used in design standards 
account for most of these errors. The exception is idealizing a structure that behaves between two extremes (e.g., semi-rigid connections, semi-flexible diaphragms). These exceptions, however, can be addressed by performing analysis on the two extremes and designing based on the most severe conditions.

2. Assumptions inherent to the analysis method or design equations. Every analysis method and design equation incorporates some assumptions. An example is the expression for shear capacity of a reinforced concrete beam without stirrups; that equation is an empirical lower bound based on laboratory tests. The load and strength reduction factors used in design standards account for these errors as well.

3. Roundoff error. Every calculation, hand or computer generated, is subjected to some roundoff. With 16-bit arithmetic being standard on desktop computers, these errors tend to be extremely small and are routinely ignored.

4. Human error. This broad category includes any error that is unintentional or unanticipated. These errors can occur at any time in the design process from design development to review of shop drawings.

Current design standards account for the first three types of errors (with a few exceptions as indicated in Idealization). The standards do not, however, account for human errors. It is the responsibility of the designer to ensure that human errors do not reduce the safety or impair the performance of a structure. Therefore, this study focuses on methods for evaluating results to ensure they are free of human errors, not all errors.

\section{Categories of Method for Evaluation}

Practicing structural engineers use a wide variety of methods to evaluate the reasonableness of both structural analysis and design results. The methods can be divided into six categories: comparisons, rules of thumb, visualization, previous experience, field, and other.

1. Comparisons. This category involves comparing two or more approaches or situations (e.g., hand calculations versus computer results, results from two different computer programs, results considering different conditions).

2. Rules of Thumb. This category involves use of simple formulas to predict member sizes or properties.

3. Visualization. This refers to visualizing the load path. Typically it involves making cuts and confirming that forces have a continuous path to the foundation.

4. Previous Experience. These strategies can only be developed through experience. They typically involve recognizing a situation as similar to previous projects.

5. Field. This is one of the least preferred strategies. It means that the problem is discovered during or after construction. 
6. Other. This category includes all of the other strategies used to discover a problem. Many of them are procedural (e.g., check model code used in the jurisdiction).

The methods contained in these categories are used throughout the analysis and design process by practicing engineers. Therefore, only some are pertinent to evaluating structural analysis results. The methods that have been incorporated into the undergraduate structural analysis course are described in more detail in this paper.

\section{Practitioner Interviews}

Before the author could begin developing new course materials for evaluating the reasonableness of results, he needed to determine how practicing engineers actually make these evaluations. In order to gather that information, the author interviewed 35 practicing structural engineers whose experiences range from 1 to 55 years, with a median of 8 years. The interviews produced 67 instances where the engineer remembered finding something unreasonable in structural analysis or design results. The interviews also produced 20 methods for finding "common" problems in results. Table 1 shows how the various methods are divided into the categories described previously.

Table 1. Summary of results of interviews with practicing structural engineers to determine methods they use to evaluate the reasonableness of structural analysis and design results.

\begin{tabular}{lc}
\hline \multicolumn{1}{c}{$\begin{array}{c}\text { Category of } \\
\text { Evaluation Method }\end{array}$} & $\begin{array}{c}\text { Number of } \\
\text { Incidents Reported }\end{array}$ \\
\hline Comparisons & 23 \\
Rules of Thumb & 7 \\
Visualization & 5 \\
Previous Experience & 22 \\
Field & 14 \\
Other & 14 \\
Not Identified & 2 \\
\hline
\end{tabular}

\section{Impact on Syllabus}

A common concern among faculty is that incorporating new material in a course often means something must be removed. Ideally, incorporating the methods for evaluating reasonableness of results into the new syllabus would not significantly alter the topics covered in the original course syllabus. The course is only ten weeks long (quarter system) and is the only required structural analysis course in the curriculum. Therefore, eliminating any existing topics would possibly leave students under prepared for subsequent coursework in structural design.

The author was able to incorporate the new material by adding evaluation to the example problems already used in the course, and by beginning most class sessions with "challenge" problems. The methods used are described in more detail in the next section. The resulting impact on the course syllabus is shown in Table 2. The same topics were taught each term. 
Only two fewer days were spent on instruction on "Force Method for Indeterminate Structures." One additional day of instruction was then allocated to "Analysis of Determinate Structures" and one to "Displacements" in order to incorporate the new material.

Table 2. Comparison of course syllabi for editions of the course before the added material (Fall 2004) and with addition of instruction on evaluating results (Fall 2005).

\begin{tabular}{lcc}
\hline \multicolumn{1}{c}{ Topic } & Number of Lectures \\
& Fall 2004 & Fall 2005 \\
\hline Structural Design Process & 1 & 1 \\
Loads \& Load Combinations & 2 & 2 \\
Idealization of Structure & 3 & 3 \\
Analysis of Determinate Structures & 2 & 3 \\
Influence Lines & 4 & 4 \\
Internal Force Diagrams & 4 & 4 \\
Approximate Analysis of Indeterminate Structures & 7 & 7 \\
Introduction to Computer Aided Analysis & 4 & 4 \\
Displacements & 6 & 7 \\
Force Method for Indeterminate Structures & 4 & 2 \\
Exams & 3 & 3 \\
\hline Total & 40 & 40 \\
\hline
\end{tabular}

Methods for Assessing the Reasonableness of Results as Taught in Undergraduate Course The methods taught in the undergraduate structural analysis course can be grouped into three types: simplified loading conditions, features of graphical answers, and approximate analysis.

\section{Simplified Loading Conditions}

In practice, many situations arise where a determinate member is loaded with a complicated combination of distributed loads and/or point loads. Undergraduates can find solutions to these problems, but such solutions are prone to human errors due to the complexity of the loading. Therefore, the author teaches the students to simplify the loading to a uniformly distributed load, a single point load, or a set of equal point loads. The simplified problem is quickly solved, thus producing an answer that can be used to evaluate the reasonableness of the answer for the actual loading conditions. This method falls into Category 1: Comparisons. An example is presented in Figure 1. An important part of the instruction includes determining whether the simplification should over- or under-estimate the solution to the problem with the complicated loading.

\section{Features of Graphical Answers}

Displaced shapes, internal force diagrams and influence lines are all graphical representations of information about a structure. Because they are graphical in nature, they inherently contain a significant amount of information. Therefore, students can be taught to anticipate certain features of these graphs. This method also falls into Category 1: Comparisons, even though the comparisons are qualitative. An example for a determinate beam is presented in Figure 2. 
Situation: The roof shown experiences snow load with drifting adjacent to the AC unit. The resulting distributed load on member AB is shown.
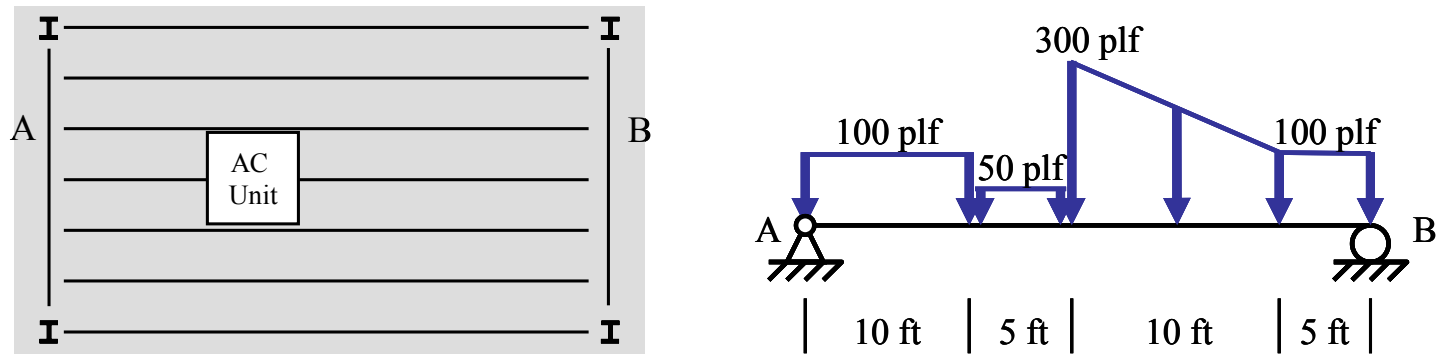

Plan View

Find: Find, approximately, the peak moment and shear experienced by member AB.

Solution:

Calculate total load applied to the member and convert it to a uniformly distributed load.

$$
\begin{aligned}
& P=100 \text { plf }(10 \mathrm{ft})+50 \mathrm{plf}(5 \mathrm{ft})+1 / 2(200 \mathrm{plf})(10 \mathrm{ft})+100 \mathrm{plf}(15 \mathrm{ft})=3750 \mathrm{lb}=3.75 \mathrm{k} \\
& \omega_{\text {avg }}=P / L=3.75 \mathrm{k} / 30 \mathrm{ft}=0.125 \mathrm{klf}
\end{aligned}
$$

Calculate $V_{\max }$ and $M_{\max }$.

$$
\begin{aligned}
& V_{\text {max }}=\omega_{\text {avg }} L / 2=0.125 \mathrm{klf}(30 \mathrm{ft}) / 2=1.88 \mathrm{k} \\
& M_{\text {max }}=\omega_{\text {avg }} \mathrm{L}^{2} / 8=0.125 \mathrm{klf}(30 \mathrm{ft})^{2} / 8=14.1 \mathrm{k} * \mathrm{ft}
\end{aligned}
$$

Anticipated variation:

$V_{\max }$ should be under-predicted since loading is lopsided, so one side will have a larger reaction.

$M_{\max }$ should be under-predicted since moving load farther from the middle of the member.

\section{Comparison with More Accurate Solution:}

Actual $V_{\max }$ and $M_{\max }$ values: $\quad$ Effect of using approximation:

$$
\begin{array}{ll}
V_{\max }=2.01 \mathrm{k} & V_{\max } \text { under-predicted by } 6.5 \% \\
M_{\max }=16.0 \mathrm{k} * \mathrm{ft} & M_{\max } \text { under-predicted by } 11.9 \%
\end{array}
$$

Figure 1. Example used in class to demonstrate how simplifying the applied load can give a quick answer that can be used to evaluate the reasonableness of a solution. 
Situation: A simply supported beam with a cantilevered end experiences uniform distributed load.

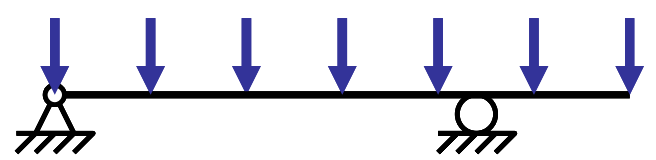

Find: Shape of the moment diagram.

\section{Solution:}

Since beam experiences uniform distributed load, expect diagram to be quadratic in all parts. Use deflected shape to provide additional clues.

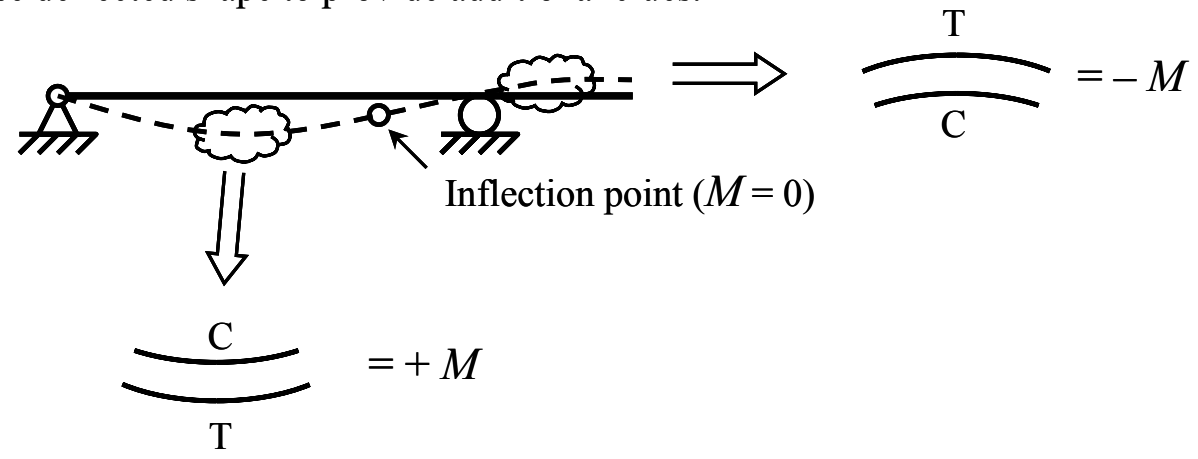

Resulting moment diagram:

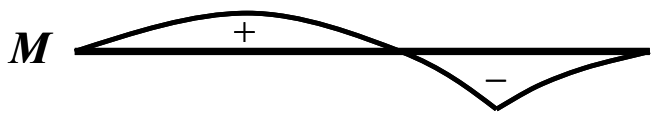

Figure 2. Example used in class to demonstrate how intuition about deflected shape helps one anticipate features of the moment diagram.

Approximate Analysis

Approximate analysis methods are commonly taught in undergraduate structural analysis courses. The Fall 2004 edition of the course included methods for rigid frames subjected to gravity loads and the portal and cantilever methods for rigid frames subjected to lateral loads. The Fall 2005 edition, however, also included instruction on methods for indeterminate trusses and braced frames. During the part of the course on computer aided analysis (see Table 2), students used the approximate methods to verify the reasonableness of results from computer analysis software. An example homework problem is provided in Figure 3. 
Situation: A two story chevron braced frame experiences the loads shown.

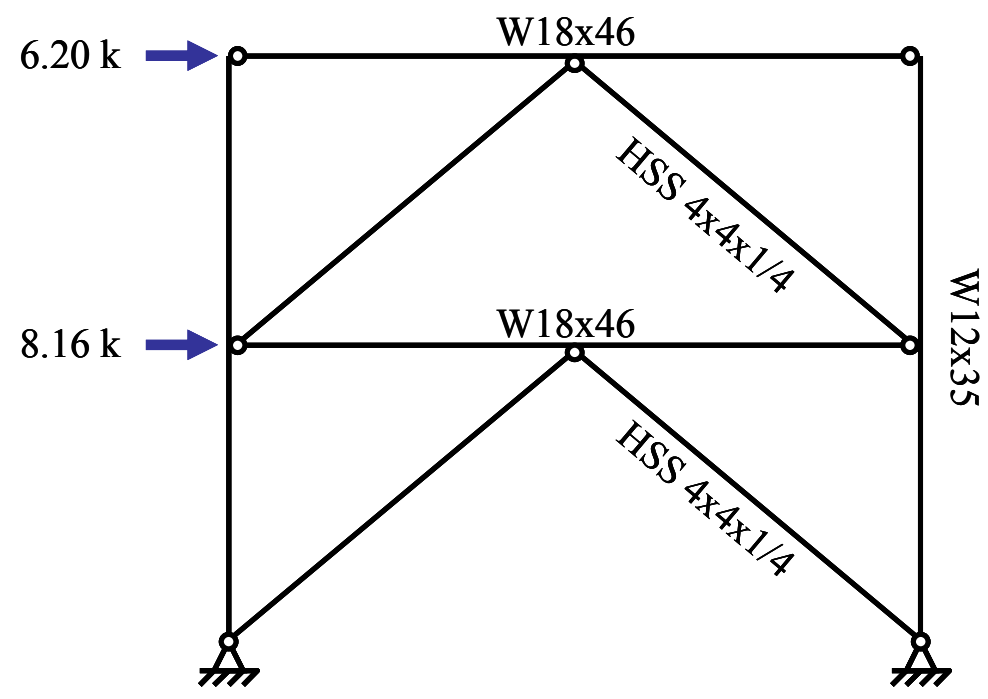

\section{Find:}

1. Using approximate analysis, determine the following unfactored internal forces:
a. Peak shear in top beam.
b. Peak moment in top beam.
c. Peak axial in top brace.
d. Peak axial in bottom brace.
e. Peak axial in bottom column.

2. Using a structural analysis program, determine the same internal forces.

3. Calculate the $\%$ difference between the computer and approximate analysis ( [approximate - computer] / computer ).

4. Based on your results in Part 3, assess whether your computer results are reasonable.

Figure 3. Homework problem designed to have students use approximate analysis methods to assess the reasonableness of computer generated solutions.

\section{Results}

To determine the impact of the new course materials, the author gathered data from exit surveys and final exams. The surveys show that students believed in the existence of reasonable answers and the importance of evaluating answers for reasonableness before changes were made in the course (Table 3). The addition of instruction on methods for evaluating the reasonableness of analysis results did not improve the students' self confidence. However, the self evaluation by the students is not necessarily a reliable indicator of ability. 
Table 3. Summary of mean scores from exit surveys in the undergraduate structural analysis course before including instruction on evaluating results (Fall 2004) and with the instruction (Fall 2005). Items were rated on a scale of 1 to 5 with 5 being the highest.

\begin{tabular}{lcc}
\hline \multicolumn{1}{c}{ Exit Survey Item } & Fall 2004 Fall 2005 \\
\hline $\begin{array}{l}\text { - Believe that a reasonable answer can be } \\
\text { obtained for most civil engineering problems }\end{array}$ & 4.4 & 4.6 \\
- Believe that undergraduates should be able to & & \\
$\begin{array}{l}\text { evaluate reasonableness of solutions } \\
\text { - Self evaluation of ability to evaluate }\end{array}$ & 4.2 & 4.3 \\
\begin{tabular}{l} 
reasonableness of solutions \\
\hline
\end{tabular}
\end{tabular}

The author used identical final exams in order to evaluate students' abilities to evaluate the reasonableness of structural analysis results. The exam was not returned in order to maintain the integrity of the exam. The exam consisted of six multiple choice questions. Within 30 minutes, students needed to determine which answer was the most reasonable and had to justify why either that answer was reasonable or why the other answers were not reasonable. The results are presented in Table 4. The correctness score is based strictly on whether the chosen answer was the most reasonable. This score can be impacted by blind guessing. The justification score is not effected by guessing; points were only awarded for valid explanations of why an answer was or was not reasonable.

Table 4. Summary of final exam performance.

\begin{tabular}{lccc}
\hline \multicolumn{1}{c}{ Group } & Number & $\begin{array}{c}\text { Correctness } \\
(\%)\end{array}$ & $\begin{array}{c}\text { Justification } \\
(\%)\end{array}$ \\
\hline Fall 2004 (no instruction) & 34 & 50 & 49 \\
Fall 2005 (with instruction) & 48 & 67 & 70 \\
Practicing Structural Engrs & 8 & 92 & 72 \\
\hline
\end{tabular}

The addition of instruction on methods for evaluating the reasonableness of structural analysis results had a measurable impact on student performance. Student ability to select the most reasonable answer and to justify its selection each increased approximately 20 percentage points. For comparison, Table 4 includes results from eight practicing structural engineers with less than 1 to over 30 years of experience with a median of 5 years. All but one of the practicing engineers had a masters degree. The practicing structural engineers averaged $92 \%$ when selecting the most reasonable answer. However, they averaged only $72 \%$ when articulating how they selected the most reasonable answer. Therefore, the additional instruction enabled students to articulate how they selected the most reasonable answer as well as practicing structural engineers, even though they are not yet able to choose the most reasonable answer as well as practicing engineers. 


\section{Conclusions}

It is the responsibility of the designer to ensure that human errors in structural analysis results do not reduce the safety or impair the performance of a structure. But to correct those errors, the designer must recognize the presence of the errors. Therefore, this study is exploring the viability of teaching undergraduate civil engineering students how to evaluate the reasonableness of structural analysis results. The addition of instruction on how to evaluate reasonableness resulted in little change in the syllabus, but caused a measurable improvement in student ability to evaluate structural analysis results.

\section{Future Work}

This investigation includes also developing course materials in order to teach more evaluation methods in the advanced structural analysis course. Once implemented, data will be available for that research as well. The NSF project will continue for one more year during which the author will revise the course materials and will continue to assess the impact of those materials on student ability to evaluate reasonableness.

To further assist students in developing the skills necessary to evaluate the reasonableness of structural analysis results, the author will be implementing instruction on metacognition in the introductory and advanced structural analysis courses. Metacognition is a sequence of steps followed by a person to monitor and improve that person's own cognitive performance in an area. Metacognition has been used to improve student learning in reading, math and science for over twenty years. Metacognition will allow students to monitor their own decision making processes as they learn how to implement the cognitive strategies.

\section{Acknowledgements}

This study was made possible by the National Science Foundation, grant no. DUE-0341212. The author would like to thank the many engineers who participated in the interviews from the following firms: Applied Technology Corporation; Arup; Burns Engineering; Leslie E. Robertson Associates, R.L.L.P.; McComas Engineering, Inc.; MMS-A/E; Skidmore, Owings \& Merrill LLP; Spencer Engineering, Inc.; and Thorton-Thomasetti Group. 